\section{AB0992 A DIFFICULT DISTINCTION - ERDHEIM-CHESTER DISEASE MIMICKING IGG4-RELATED DISEASE}

P. Korsten ${ }^{1}$, C.O. Sahlmann ${ }^{2}$, S. Hakroush ${ }^{3} .{ }^{1}$ Department of Nephrology and Rheumatology; ${ }^{2}$ Department of Nuclear Medicine; ${ }^{3}$ Institute of Pathology, University Medical Center Göttingen, Göttingen, Germany

Background: Rheumatologists are often confronted with systematic and rare diseases. Even among the rare diseases, clinical features may overlap, but treatment options for different diseases may differ.

Objectives: To illustrate the diagnostic challenges the clinician is confronted with when facing rare and systemic diseases in clinical practice with a case report where clinical features were overlapping.

Methods: A 64-year-old woman was presented to our institution after suffering from two ischemic strokes. Cerebral imaging was suggestive of large-vessel vasculitis. Additionally, imaging also revealed a left-sided retro-orbital mass. Her past medical history was notable for retroperitoneal fibrosis with ureteral obstruction requiring percutaneous drainage six months before presentation and placement of a cardiac pacemaker several years ago. Treatment with high-dose prednisone had been initiated but showed no clinical or laboratory improvement. Results: Clinical examination revealed mild persistent facial hemiparesis and difficulty with ambulation. Mild exophthalmos was present on her left side. On palpation, there were palpable axillary masses bilaterally. Otherwise, her physical examination was normal. Laboratory analysis revealed elevated soluble interleukin-2 receptor levels at $2800 \mathrm{IU} / \mathrm{mL}(\mathrm{N}: 230-770 \mathrm{IU} / \mathrm{mL})$ and mildly increased C-reactive protein at $10 \mathrm{mg} / \mathrm{L}(\mathrm{N}:<5 \mathrm{mg} / \mathrm{L})$. Autoantibodies, including rheumatoid factor, IgG4, ANCA, ANA, and ENA were all within normal range. Plasma cells were normal on flow cytometric analysis. A PET-CT was performed which revealed signs of alveolitis, retroperitoneal fibrosis, axillary masses with strong tracer uptake (figure 1) as well as tracer uptake of the left femur and periaortic sheathing. We performed a biopsy of the left-sided axillary mass. Histopathologic analysis showed Touton giant cells and septal fibrosis. Staining was negative for CD1a and S100 but positive for CD68. Molecular pathologic analysis revealed the presence of the BRAFV600E mutation, findings that are consistent with the diagnosis of Erdheim-Chester disease. We initiated treatment with the BRAF-inhibitor vemurafenib and will follow the patient closely. Staining for lgG4 was negative.
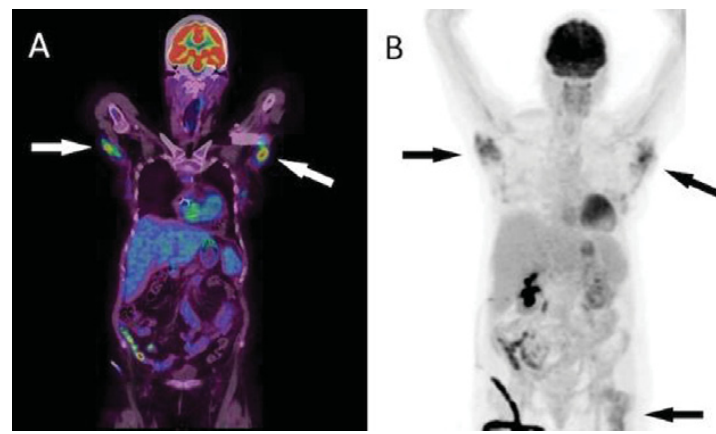

Conclusions: Erdheim-Chester disease (ECD) and IgG4-related disease are very rare disorders and can present with similar clinical findings, such as retroperitoneal fibrosis, alveolitis or lymph node enlargement. About 500 cases of ECD worldwide have been reported. ECD is a non-Langerhans cell histiocytosis with a reported five-year survival rate of about $68 \%$. Roughly $50 \%$ of patients show a BRAF mutation. Various treatment options have been reported and are recommended by the recently published consensus guidelines (1). These include interferon-alpha, vemurafenib and also TNF-alpha inhibitors, such as infliximab, but also interleukin-antagonists, such as anakinra (IL-1) or tocilizumab (IL-6). Rheumatologists have to consider non-Langerhans cell histiocytoses in the differential of systemic diseases because the distinction can be difficult and imaging, as well as meticulous histopathologic analyses, are necessary to correctly diagnose these patients because treatment differs.

References:

[1] Diamond EL et al. Consensus guidelines for the diagnosis and clinical management of Erdheim-Chester disease. Blood 2014 Jul 24;124(4):483-92. Disclosure of Interest: None declared

DOI: 10.1136/annrheumdis-2017-eular.6903

\title{
AB0993 ELDERLY - ONSET SARCOIDOSIS: A SINGLE CENTER COMPARATIVE STUDY
}

S. Kobak ${ }^{1}$, F. Sever ${ }^{2}$, H. Semiz ${ }^{3}$, M. Orman ${ }^{4} \cdot{ }^{1}$ Rheumatology, Istinye University Faculty of Medicine, LIV Hospital, Istanbul; ${ }^{2}$ Chest Diseases, Medicalpark Hospital; ${ }^{3}$ Internal Medicine; ${ }^{4}$ Statistics, Ege University Faculty of Medicine, Izmir, Turkey

Background: Sarcoidosis is a chronic granulomatous inflamatory disease characterized with non-caseified granuloma formation. It is rarely affects patients older than 65 years old.

Objectives: The purpose of this study is to compare and evaluate the demografic, clinical and laboratory features of elderly-onset (EOS) and young-onset sarcoidosis (YOS) patients.

Methods: One hundred and thirty one patients diagnosed with sarcoidosis according to clinical, radiologic and histopathological evaluation were included in this study. The patients with initial symptoms started after age 65 were accepted as EOS. Demografic, clinic, radiologic, and laboratory data and the medication which the patients recieved were recorded and retrospectively evaluated.

Results: Twenty $(15.3 \%)$ of 131 patients were diagnosed as EOS, and 111 $(84.7 \%)$ patients were evaluated as YOS. Fifteen of $20 \mathrm{EOS}$ patients were female and 5 of them were male. Average duration of the disease was determined as 38.4 months for YOS and 22.5months for EOS ( $p=0.556)$. Delay of the diagnosis was 12 months for YOS while it was 3months for EOS $(p=0.001)$. Higher rates of fatique, comorbid diseases and more Hydroxychloroquine ( $\mathrm{HQ}$ ) use were detected in EOS patients comparing to YOS $(p=0.010, p=0.003$ and $p=0.039$ respectively). There was obviously more disease modifying anti-rheumatic drugs (DMARDs) use by YOS group but statistical difference wasn't significant. The 3-year survival rate after diagnosis of sarcoidosis was \%95 in the EOS group, compared with $\% 100$ in the YOS group.

Conclusions: In this study we showed that YOS and EOS patients may be presented with different clinical, and laboratory features. EOS patients are characterized with higher rates of fatique and comorbid diseases, less inflammatory sign and delayed diagnosis, and less DMARDs usage.

References:

[1] Newman LS, Rose CS, Maier LA. Sarcoidosis. N Engl J Med 1997; 336: $1224 \pm 1234$.

[2] Chen ES, Moller DR. Etiology of sarcoidosis. Clin Chest Med. 2008; 29:365377.

[3] lannuzzi MC, Rybicki BA, Teirstein AS. Sarcoidosis. N Engl J Med. 2007;357:2153-65.

[4] lannuzzi MC, Fontana JR. Sarcoidosis: clinical presentation, immunopathogenesis, and therapeutics. J Am Med Assoc. 2011;305:391-9.

[5] Baughman RP, Teirstein AS, Judson MA, Rossman MD, Yeager H Jr, Bresnitz EA, et al. Clinical characteristics of patients in a case control study of sarcoidosis. Am J Respir Crit Care Med.2001;164:1885-9.

Disclosure of Interest: None declared

DOI: 10.1136/annrheumdis-2017-eular.1377

\section{AB0994 ANTI TNF-ALPHA THERAPY WOULD BE LIFESAVING IN DEFICIENCY OF ADENOSINE DEAMINASE-2}

S. Sahin ${ }^{1}$, A. Adrovic ${ }^{1}$, K. Barut ${ }^{1}$, S. Ugurlu ${ }^{2}$, E.T. Turanli ${ }^{3}$, H. Ozdogan ${ }^{2}$, O. Kasapcopur ${ }^{1}$. ${ }^{1}$ Department of Pediatric Rheumatology; ${ }^{2}$ Department of Rheumatology, Istanbul University, Cerrahpasa Medical Faculty; ${ }^{3}$ Department of Molecular Biology and Genetics, Istanbul Technical University, Faculty of Science and Letters, Istanbul, Turkey

Background: Deficiency of adenosine deaminase-2 (DADA2) is a rare form of autoinflammatory disorder with limited reported cases.

Objectives: In this study, we have characterized the clinico-immunological, radiological and genetic characteristics of eight childhood-onset DADA2 patients. We aimed to compare these features between surviving and deceased patients. Methods: Demographic features, clinical characteristics, imaging findings, mutations and pharmacological treatments compared between surviving and deceased DADA2 patients.

Results: Eight patients from seven families were enrolled. While five of them were still surviving, three of them had died due to various reasons. Median age of the patients at disease onset and diagnosis were 7 years (range $0.5-13$ years) and 14 years (range 5-27years), respectively. The main clinical manifestations were cutaneous manifestations (7/8), recurrent low-grade fever (6/8), neurological involvement (6/8) and gastrointestinal involvement (5/8). All patients had increased acute phase reactants at presentation and also during flares. Until the diagnosis of DADA2, five patients have been followed-up with the diagnosis of PAN; two patients both with PAN and FMF, and one patient with CAPS and vasculitis.

Abstract AB0994 - Table 1. Clinical features and imaging results of DADA2 patients

\begin{tabular}{|c|c|c|c|c|c|c|c|c|}
\hline Age at disease onset & 7 & 0.5 & 1 & 9 & 7 & 7 & 1.5 & 13 \\
\hline Previous diagnosis & PAN & PAN & PAN & PAN & PAN-FMF & PAN-FMF & CAPS, Vasculitis & PAN \\
\hline GIS involvement & Yes & Yes & No & Yes & Yes & Yes & No & No \\
\hline Cutaneous findings & Yes & Yes & Yes & Yes & Yes & Yes & Yes & No \\
\hline Neurologic involvement & Yes & Yes & Yes & Yes & No & No & Yes & Yes \\
\hline Neuroimaging findings & Ischemic stroke & Hemorrhagic stroke & Periventricular leucomalasia & Normal & Normal & Cerebral atrophy & İschemic stroke & İschemic stroke \\
\hline CECR1 & p.Gly47Arg & p.Gly47Arg & p.Gly47Arg & p.Gly47Val and p.Trp264Ser & p.Gly47Arg & p.Gly47Arg & p.Gly47Arg and p.Arg306Ter & p.Gly47Arg \\
\hline
\end{tabular}

